\title{
DESARROLLO Y VALIDACIÓN DE LA ESCALA DE EXPECTATIVAS DE RECHAZO SOCIAL (ERS)
}

\author{
Valeria Estefania Morán ${ }^{1}{ }^{\mathbb{D}}$, \& Fabian Orlando Olaz \\ Consejo Nacional de Investigaciones Científicas y Técnicas, Córdoba, \\ Argentina; Universidad Nacional de Córdoba, Córdoba, Argentina. ${ }^{2}$
}

\begin{abstract}
RESUMEN
El desempeño social de los individuos se ve influido por las expectativas que las personas tienen sobre el resultado de su conducta, ya que estas permiten anticipar el futuro y planificar cursos de acción. Las expectativas de rechazo social han sido definidas como pensamientos que anticipan la posibilidad de ser rechazado en una situación interpersonal y cuando son muy frecuentes pueden traer aparejado un sinnúmero de dificultades en la vida social de las personas. El objetivo de este estudio fue construir la Escala de Expectativas de Rechazo Social (ERS) para población adulta. En primer lugar, se confeccionaron 51 ítems preliminares que fueron sometidos a juicio de expertos $(\mathrm{N}=6)$ para obtener evidencia de validez de contenido. En segundo lugar, utilizando muestreo accidental, se llevó a cabo un análisis factorial exploratorio $(\mathrm{N}=320)$ y confirmatorio $(\mathrm{N}=458)$. Como resultado se obtuvo una escala final compuesta por 27 ítems distribuidos en tres factores interpretados como Expectativas de rechazo ante situaciones con vínculos establecidos, Expectativas de rechazo en situaciones de establecimiento de nuevos vínculos y Expectativas de rechazo en lugares públicos y ante desconocidos. Se evaluó la consistencia interna de la escala, obteniendo coeficientes muy buenos y excelentes para cada factor (de .71 a .88). Finalmente, se obtuvieron correlaciones positivas significativas entre las escalas del test y diferentes medidas de ansiedad social. Se puede concluir que la ERS es un instrumento válido y confiable para la evaluación de las expectativas de rechazo social en población adulta, resultando una herramienta de utilidad en investigación y diagnóstico.
\end{abstract}

Palabras Claves

expectativas de rechazo social; desempeño interpersonal; adultos argentinos

\begin{abstract}
The social performance of individuals is influenced by the expectations that people have about the outcome of their behavior, since these allow us to anticipate the future and plan courses of action. The expectations of social rejection have been defined as thoughts that anticipate the possibility of being rejected in an interpersonal situation. When they are frequent can bring a number of difficulties in the social life of people. The aim of this study was to build the Social Rejection Expectations Scale (SRE) for the adult population. First, 51 preliminary items were submitted to expert judgment $(\mathrm{N}=6)$ to obtain evidence of content validity. Second, using an accidental sampling, an exploratory $(\mathrm{N}=320)$ and confirmatory factor analysis $(\mathrm{N}=458)$ was performed. As a result, a final scale was obtained, consisting of 27 items distributed in three factors named as Expectations of rejection in situations with known people, Expectations of rejection in situations of establishment of new relationships and Expectations of rejection in public places and with unknown people. The internal consistency of the scale was evaluated, obtaining very good and excellent coefficients for each factor (from .71 to .88). Finally, significant positive correlations were obtained between the scales of the test and different measures of social anxiety. It can be concluded that the SRE is a valid and reliable instrument for the evaluation of expectations of social rejection in the adult population, being a useful tool in research and diagnosis.
\end{abstract}

\section{Keywords}

expectations of social rejection; interpersonal performance; Argentinean adults

\footnotetext{
${ }^{1}$ Correspondence about this article should be addressed to Valeria Estefania Morán: moranvaleria@gmail.com

2 Instituto de Investigaciones Psicológicas (IIPsi), de doble dependencia.
} 


\section{DEVELOPMENT AND VALIDATION OF THE SOCIAL REJECTION EXPECTATIONS SCALE (SRE)}

\section{Introducción}

En el presente, asistimos a un proceso acelerado de cambio social donde las complejas redes sociales de las cuales forma parte el individuo con sus reglas de interacción específicas, hacen que la calidad de las interacciones sociales sea especialmente valorada. Por esto, un adecuado repertorio de habilidades sociales constituye un factor de importancia en la adaptación y ajuste interpersonal, y en la salud y bienestar psicológico de las personas.

Frente a las diferentes situaciones de demanda social que enfrentamos en la vida cotidiana, un adecuado repertorio comportamental puede operar como un factor de ajuste y facilitar el afrontamiento eficaz de situaciones problemáticas. Dentro de este repertorio, existen ciertos mecanismos cognitivos a través de los cuales el hombre regula su conducta basándose en criterios internos y reacciones autoevaluadoras que permiten el ejercicio de la agencia personal (Pajares \& Olaz, 2008). Según Bandura las personas tienen la facultad de ejercer control sobre sus pensamientos, su motivación y sus acciones a través de un autosistema que permite percibir, regular y evaluar comportamientos (Olaz, 2011) utilizando información proveniente del ambiente. A su vez, este autosistema está constituido por una serie de capacidades, entre las que la predicción ocupa un lugar fundamental.

En este sentido, las expectativas de resultado que el individuo posee frente a situaciones de demanda específicas hacen referencia a las predicciones de una persona acerca de las posibles consecuencias de su comportamiento. Estas expectativas guían la selección de conductas que el individuo es capaz de emitir ante determinadas situaciones (Caballo et al., 2011). Para Bretherton y Munholland (1999), la influencia de estos modelos mentales permite a los individuos anticiparse al futuro y hacer planes, sirviendo de guía en la valoración de las situaciones sociales y en la asimilación de nuevas experiencias.

Tal como señala Olaz (2012) las expectativas de resultado afectan a los intereses y a las acciones. Cuanto más valorados sean los resultados percibidos, mayores probabilidades habrá de que un individuo se interese y adopte cursos de acción determinados. Asimismo, si una persona espera resultados negativos ante una interacción social, esto provocará que disminuya su interés y compromiso en estas actividades, lo 
cual puede llevar a que efectivamente tenga un desempeño deficitario o que evite próximas exposiciones, impidiendo el aprendizaje o adquisición de habilidades, perpetuando de esta manera estas creencias.

Las expectativas de rechazo social son pensamientos que anticipan la posibilidad de ser rechazado en una situación interpersonal (Morán et al., 2018a). El deseo de lograr la aceptación y prevenir el rechazo es ampliamente reconocido como un factor fundamental en las relaciones interpersonales (Tévez Carranza et al., 2018). Leary (2001, 2005, citado en Leary et al., 2006) conceptualiza el rechazo en términos de un continuo sobre el cual los individuos califican sus relaciones en diferentes grados. De esta forma, se considera rechazo cuando la persona evalúa su relación con otro individuo como insignificante o sin importancia. En este sentido, una persona con expectativas de rechazo social piensa que los demás no valorarán la interacción con ella tanto como le gustaría (Smart Richman \& Leary, 2009). Esto genera un impacto en el afrontamiento y desempeño ante las situaciones de interacción social donde estas expectativas se activan.

En diferentes investigaciones se ha observado que las personas que piensan que van a ser capaces de generar las impresiones deseadas en los demás tienen una autoeficacia social más alta y se sienten menos ansiosas en encuentros interpersonales (Leary, 1986; Lee \& \& Hayes-Skelton, 2017). Por otro lado, Bellack (1979 en Caballo et al 2011) señala que es posible que conductas socialmente habilidosas sean inhibidas debido a que son consideradas inapropiadas o generadoras de resultados negativos. Siguiendo esta línea, Fiedler y Beach (1978) demostraron que actuar asertivamente se ve sustancialmente afectado por la predicción que la persona tiene sobre su desempeño y sus consecuencias.

Estos sucesos negativos impactan en la vida social de las personas y consecuentemente en su bienestar. Algunas investigaciones (Tabak et al., 2016; MacDonald \& Leary, 2005; Wirth et al., 2015) afirman que la pérdida o potencial pérdida de una conexión conllevan un sufrimiento social para el individuo, tanto fisiológico como afectivo.

Las expectativas, como otras cogniciones sociales, responden al principio de activación cognitiva, según el cual las cogniciones deben ser activadas para que tengan alguna influencia en el procesamiento de información. Esto ocurre en función de varios factores como la información sobresaliente de la situación, tendencias personales, priming, emociones, metas, activación de otros pensamientos, etc (Baldwin \& Dandeneau, 2006). 
$\mathrm{Al}$ respecto, Wirth et al. (2015) llevaron a cabo un estudio con el fin de observar si las señales provenientes del contexto social evocan expectativas de rechazo social. Los resultados demostraron que cuando los individuos esperan ser rechazados sobre la base de señales sociales que anticipan el rechazo, los efectos del rechazo efectivo sobre los niveles de satisfacción de necesidades básicas, sentimientos negativos y de exclusión, suelen ser menores, aunque no eliminados por completo, a los de aquellos individuos que no anticipan el rechazo por haber recibido señales de aceptación del entorno. Por otro lado, el malestar asociado a la expectativa de rechazo era compensado cuando la persona era incluida en el grupo. Por otro lado, los resultados sugieren que las expectativas de inclusión no cumplidas acentúan las consecuencias aversivas del rechazo. Los autores sostienen que esto es consecuente con la función adaptativa que cumplen los sistemas de dolor social en la supervivencia, por lo que la incapacidad de estar alerta ante un rechazo puede ser devastador.

Estos resultados muestran la fuerte tendencia de las personas a dirigir su atención y aprender de contingencias sociales de aceptación y rechazo interpersonal, incluso en pocos ensayos. Asimismo, se observa el poderoso impacto de las cogniciones relacionales temporarias sobre percepciones, emociones y conductas interpersonales, lo cual pone en cuestión el supuesto de que existen rasgos interpersonales inmutables adquiridos tempranamente en la vida y luego expresado inevitablemente en todas las relaciones.

Como se ha expuesto hasta aquí, las expectativas de rechazo social constituyen pensamientos de fácil adquisición y de gran impacto en la conducta, autoestima, afectividad y creencias de las personas (Caouette \& Guyer, 2016; Driscoll et al., 2017; Tobia et al., 2017). Creer que uno es querido, apreciado, incluido y valorado fomenta el bienestar, la autoestima y la confianza, mientras que la sensación de no ser aceptado, no apreciado, excluido o desvalorizado evoca emociones negativas y puede dar lugar a reacciones antisociales como la agresividad o la retirada.

Downey et al (1994) afirman que las personas con sensibilidad al rechazo lo esperan ansiosamente, lo perciben fácilmente y reaccionan de forma exagerada ante el mismo. En este sentido, las personas con alta sensibilidad al rechazo están preocupadas por la posibilidad de ser rechazadas a la vez que esperan el rechazo en lugar de la aceptación. Por el contrario, las personas que tienen baja sensibilidad al rechazo, muestran niveles más bajos de preocupación por la posibilidad de ser rechazadas, y esperan la aceptación de los otros significativos (Ayduk et al., 2001). Downey y Feldman (1996) proporcionaron evidencia experimental que sustenta que las personas que esperan el 
rechazo, y son altamente sensibles a él, son capaces de percibirlo fácilmente, aun cuando el comportamiento sea ambiguo. Esto genera malestar y baja satisfacción en los vínculos sociales que estos individuos establecen.

En este punto, si bien es posible encontrar evidencias y postulados teóricos sobre cómo las expectativas de resultado negativas se vinculan con diferentes problemas psicológicos, no se reportan instrumentos de evaluación que midan expectativas de rechazo social específicamente. Sumado a esto, en los estudios donde se estudia este constructo (por ejemplo, Wirth et al., 2015) no se trabaja con instrumentos que cumplan con los criterios psicométricos recomendados por la literatura especializada (AERA et al., 2014). Teniendo en cuenta lo mencionado, en el presente trabajo se presenta la construcción de la Escala de Expectativas de Rechazo Social (ERS) para adultos.

\section{Método}

\section{Participantes}

Para el estudio de análisis factorial exploratorio se trabajó con una muestra inicial de 320 participantes (73.7\% mujeres, $26.3 \%$ hombres), de edades comprendidas entre 18 y 70 años de edad $(\mathrm{M}=25.03 \mathrm{~s}=7.92)$. Para el análisis factorial confirmatorio la muestra se conformó con 458 participantes (75.3\% mujeres, $24.7 \%$ hombres), de edades comprendidas entre 18 y 70 años de edad $(M=25.5, s=7.81)$. El muestreo utilizado fue no probabilístico de tipo accidental.

\section{Instrumentos}

Test de Ansiedad Social para estudiantes universitarios, TAS-U (Moran et al., 2018b): Este instrumento fue construido para estudiantes universitarios argentinos. Utilizando una escala de 1 a 10 ( 1 nada - 10 mucho), la persona debe indicar la intensidad con que experimenta ansiedad, nerviosismo o malestar ante determinadas situaciones sociales ("Responder a la pregunta de un profesor en clase", por ejemplo). El instrumento se compone de 27 ítems agrupados en cuatro factores obtenidos mediante AFE y AFC, denominados Ansiedad ante situaciones sociales con personas conocidas, compuesto por 11 ítems $(\omega=.86)$, Ansiedad ante situaciones de desempeño académico o laboral compuesto por siete ítems $(\omega=.88)$, Ansiedad a ser observado por otros en situaciones generales, compuesto por seis ítems $(\omega=.77)$ y Ansiedad ante situaciones de abordaje afectivo o sexual, compuesta por cuatro ítems $(\omega=.81)$. 


\section{Procedimiento}

Los participantes fueron informados acerca del propósito del estudio previo a la participación, y firmaron un formulario de consentimiento que describe el objetivo, la voluntariedad de su participación y la naturaleza confidencial de los datos. Los cuestionarios se respondieron vía online.

Previo a cada análisis, se evaluó la calidad de todas las bases de datos, analizando valores perdidos y casos atípicos univariados y multivariados.

\section{Resultados}

\section{Diseño y redacción de ítems}

Para el diseño de los ítems preliminares se revisaron un total de 9 escalas que evaluaban expectativas de resultado en relación a diferentes dominios de comportamiento, utilizadas en diferentes países y para diferentes poblaciones. Esta revisión tuvo como objetivo determinar el estilo de redacción para este tipo de enunciados, atendiendo a las consideraciones de Lent y Brown (2006) para la construcción de instrumentos de medición de constructos basados en la Teoría Social Cognitiva. Dado que no se encontró en la literatura escalas que evaluasen expectativas de rechazo social, se revisaron instrumentos de evaluación de constructos relacionados, tales como miedo a la evaluación negativa (Leary, 1983), sensibilidad al rechazo (Mehrabian, 1970, 1976) y miedo al rechazo (Sokolowski, et al., 2000) para identificar dimensiones comunes y situaciones específicas de rechazo abordadas en cada uno de sus ítems. Por otro lado, también se analizaron instrumentos locales construidos para evaluar constructos referidos a relaciones interpersonales, tales como habilidades sociales (Morán et al., 2015), autoeficacia social (Olaz, 2012), los cuales fueron utilizados como guía para incluir situaciones de interacción social propias de nuestro contexto.

Los ítems de la Escala de Expectativas de Rechazo Social (ERS) fueron redactados teniendo en cuenta diferentes tipos de desempeño (individual o en interacción), diversos contextos y demandas, distintos niveles de familiaridad de los participantes y también diferente número de personas en la interacción. De esta manera se redactaron 51 ítems iniciales a ser evaluados. La escala de respuesta propuesta fue de tipo Likert, utilizando un recorrido de 1 a 5, donde la persona que responde debe indicar 
la frecuencia en que piensa de la manera que cada ítem expresa ante cada situación social planteada.

\section{Evidencia de contenido}

Los ítems preliminares del instrumento fueron entregados a jueces expertos en construcción de escalas en general y en el estudio de constructos vinculados a las relaciones interpersonales. Se entregó a cada juez $(\mathrm{N}=6)$ un formato estandarizado de calificación, incluyendo los ítems y los criterios de evaluación. Los ítems fueron evaluados en función de criterios de claridad semántica, corrección sintáctica, y congruencia de cada ítem con el constructo teórico que se pretendía medir. Para ello, se solicitó a los jueces que indicaran si consideraban pertinente incluir el ítem en base a su contenido, y que, por otro lado, los calificaran en sus aspectos formales utilizando una escala de 1 a 5 donde el puntaje menor indicaba una baja claridad y una recomendación implícita para su reelaboración, mientras que puntaje mayor significaba una alta calidad formal. Finalmente, se le permitió a cada juez realizar observaciones y esclarecimientos que consideraran pertinentes para el perfeccionamiento de cada reactivo.

Para determinar el acuerdo entre jueces sobre la calidad de los ítems se utilizó el coeficiente V de Aiken. Este coeficiente se basa en la opinión de $\mathrm{N}$ jueces expertos sobre la validez de un material evaluativo, sus puntajes van desde 0 a 1 , en donde 1 indica un acuerdo perfecto entre los jueces. Sumado a ello, se utilizó el método score para el cálculo de los intervalos de confianza de cada coeficiente, debido a que no depende de la distribución normal de la variable y es altamente exacto (Merino Soto \& Livia Segovia, 2009).

En primer lugar, se consideró la indicación de los jueces acerca de la inclusión de cada ítem en la escala y las observaciones realizadas en los casos en que se sugirió la eliminación. Como resultado, se decidió eliminar cuatro ítems cuyo contenido no evaluaba expectativas de rechazo social, sino constructos relacionados.

Posteriormente, se calculó la concordancia de los jueces. De los 47 reactivos evaluados, 38 obtuvieron resultados satisfactorios, presentando coeficientes V superiores e iguales a .85 con intervalos de confianza entre $95 \%$ y $99 \%$. Por otro lado, siete ítems obtuvieron coeficientes $\mathrm{V}$ entre .84 y .70, los cuales fueron conservados por considerarse relevantes como indicadores del constructo. Finalmente, dos ítems presentaron coeficientes menores a .70 con intervalos de confianza no significativos, por lo tanto, se 
decidió eliminar estos reactivos. Se analizaron las observaciones realizadas por los expertos para los 45 ítems finales, y se realizaron modificaciones simples (signos de puntuación, corrección de sesgo de género o utilización de sinónimos, por ejemplo) en la redacción de 20 reactivos.

\section{Evidencia de estructura interna y análisis de consistencia interna}

\section{Análisis factorial exploratorio}

A partir del estudio anterior se elaboró un formato preliminar del instrumento el cual fue administrado a la muestra $(\mathrm{N}=320)$. Previo al AFE se evaluó el ajuste entre las distribuciones de cada ítem y los supuestos del análisis multivariado. Para comprobar los supuestos de normalidad de la muestra se realizaron análisis de asimetría y curtosis en cada ítem y se realizó una inspección gráfica de la distribución de los puntajes (histogramas con curva normal y gráficos Q-Q). Un último análisis consistió en el diagnóstico de multicolinealidad entre los ítems. Con este propósito, se analizaron los índices de tolerancia, las matrices de intercorrelación entre los ítems, y los índices de condición (conditioning indexes) para cada uno de los reactivos. Los resultados en todos los casos fueron satisfactorios ya que no se observaron correlaciones entre ítems iguales o mayores a .90 , los índices de tolerancia fueron superiores a .40 y los índices de condición inferiores a 30.

Como resultado del análisis de componente principales sobre los 45 ítems de la escala, se obtuvo un índice de adecuación muestral KMO (Kaiser-Meyer-Olkin) de .93, y el test de esfericidad de Bartlett fue significativo a un nivel $p<.001$, lo cual indica una adecuada íntercorrelación entre los datos y la factibilidad de realizar el análisis factorial. Utilizando la regla de Kaiser-Gutman se obtuvo una solución inicial de 10 factores iniciales con autovalores superiores a 1, los cuales explicaban en conjunto un $58.79 \%$ de la varianza total de la prueba. Por otro lado, los resultados del análisis paralelo de Horn (HPA; Horn, 1965) y del Scree test (Catell, 1966) permitieron identificar un máximo de tres factores.

Sobre la base de estos resultados se volvió a analizar los datos solicitando la extracción de diferentes soluciones hasta tres factores, utilizando Ejes Principales como método de extracción y rotación oblicua (Promax). Los resultados permitieron inferir la existencia de entre tres y cuatro factores, observándose que la solución más clara y estable fue la de tres. 
A partir de la inspección de la matriz de componentes se eliminaron los ítems con pesos factoriales menores a .40 (excepto dos casos en que los valores fueron cercanos y se consideraron indicadores relevantes del constructo), aquellos ítems con cargas compartidas con otro factor (superiores a .30), y aquellos ítems que no correlacionaban con ningún factor. De esta manera, se retuvieron un total de 32 ítems distribuidos en tres factores que explicaron un $43.18 \%$ de la varianza de respuestas al test. Tal como recomiendan Costello y Osbourne (2005) incluyeron al menos 5 ítems con cargas factoriales cercanas a .50 (ver Tabla 1). Finalmente, se evaluó la consistencia interna de la escala y sus factores utilizando el coeficiente Alfa de Cronbach. Como puede observarse en la Tabla 1 se obtuvo una adecuada fiabilidad en todos los constructos latentes, cuyos coeficientes presentaron valores considerados buenos y muy buenos.

Tabla 1.

Matriz de configuración de los 32 ítems preliminares del ERS

\begin{tabular}{|c|c|c|c|}
\hline & \multicolumn{3}{|c|}{ Factor } \\
\hline & $\begin{array}{c}1 \\
\alpha=.90\end{array}$ & $\begin{array}{c}2 \\
\alpha=.84\end{array}$ & $\begin{array}{c}3 \\
\alpha=.76\end{array}$ \\
\hline Item1 & 0.78 & & \\
\hline Item2 & 0.74 & & \\
\hline Item3 & 0.68 & & \\
\hline Item4 & 0.63 & & \\
\hline Item5 & 0.61 & & \\
\hline Item6 & 0.58 & & \\
\hline Item7 & 0.57 & & \\
\hline Item8 & 0.57 & & \\
\hline Item9 & 0.56 & & \\
\hline Item10 & 0.54 & & \\
\hline Item11 & 0.53 & & \\
\hline Item12 & 0.53 & & \\
\hline Item13 & 0.52 & & \\
\hline Item14 & 0.51 & & \\
\hline Item15 & 0.51 & & \\
\hline Item16 & 0.47 & & \\
\hline Item17 & 0.44 & & \\
\hline Item 18 & 0.42 & & \\
\hline Item19 & 0.42 & & \\
\hline Item 20 & & 0.85 & \\
\hline Item21 & & 0.69 & \\
\hline Item22 & & 0.56 & \\
\hline Item23 & & 0.53 & \\
\hline Item24 & & 0.53 & \\
\hline Item 25 & & 0.53 & \\
\hline Item 26 & & 0.53 & \\
\hline Item 27 & & & 0.90 \\
\hline Item 28 & & & 0.73 \\
\hline Item29 & & & 0.64 \\
\hline Item30 & & & 0.43 \\
\hline Item31 & & & 0.39 \\
\hline Item32 & & & 0.39 \\
\hline
\end{tabular}

El examen del contenido de los ítems permitió interpretar el factor 1 como Expectativas de rechazo en situaciones con vínculos establecidos, el factor 2 como Expectativas de rechazo en situaciones de establecimiento de nuevos vínculos, y el factor 3 como Expectativas de rechazo en lugares públicos y ante desconocidos.

\section{Análisis factorial confirmatorio y fiabilidad compuesta}

En el primer paso se especificó un modelo de acuerdo a la estructura factorial obtenida mediante AFE considerando asimismo aspectos teóricos vinculados al constructo y a otras variables interpersonales (estructuras confirmadas en otras escalas, tales como la escala de ansiedad social). Dado que el modelo contaba con más datos que parámetros a estimar, y se disponían suficientes grados de libertad (461 gl), se procedió al contraste del modelo. Se realizó el 
AFC con un procedimiento de estimación directo por medio del método de estimación máxima verosimilitud teniendo en cuenta que los datos se distribuían de una forma cercana a la normal. Los análisis de ajuste del modelo inicial presentaron un coeficiente chi-cuadrado significativo $(1288.57$; sig. $=.000)$. Por otro lado, los índices de ajuste si bien no alcanzan los valores establecidos, fueron cercanos $\mathrm{GFI}=.84, \mathrm{CFI}=.85, \mathrm{RMSEA}=$ .06 y $\mathrm{SRMR}=.06$. El análisis de CMIN/DF dio como resultado un valor de 2.79, lo cual indica que el ajuste del modelo no era óptimo. Los coeficientes de regresión estandarizados obtenidos fueron de .44 a .72 para el factor 1 , de .56 a .6 para el factor 2 , y de .41 a .72 para el factor 3 .

Tomando en consideración los resultados obtenidos se procedió a la reespecificación del modelo. En primer lugar, se omitieron aquellos ítems que presentaran covarianzas residuales estandarizadas altas (superiores a 2.58, según los criterios de Hair, Black, Babin, Anderson, \& Tatham, 2006), y se atendió a los índices de modificación (MI) de los mismos y a la importancia del ítem como indicador del constructo. Como resultado se obtuvo un modelo final de 27 indicadores agrupados en 3 factores y con coeficientes de regresión significativos (ver Tabla 2). En la re- especificación del modelo se eliminaron 5 ítems y se introdujeron covarianzas entre los errores de algunos ítems que pertenecían al mismo factor y que tenían un contenido equivalente, siguiendo las recomendaciones de Brown (2006).

En relación a los índices de ajuste, si bien se obtuvo un coeficiente chi-cuadrado significativo, su valor se redujo considerablemente $(670.62$; sig.= .000). Por otra parte, a pesar de que el valor CMIN/DF fue de 2.10, los otros índices de ajuste fueron adecuados $(\mathrm{CFI}=.91, \mathrm{GFI}=.90, \mathrm{RMSEA}=.049$ y SRMR= .047), por lo cual se conservó el modelo priorizando la retención de ítems teóricamente relevantes. Finalmente, se calculó la fiabilidad compuesta $(\omega)$ de las escalas, obteniendo .88 para el factor 1.84 para el factor 2 , y .71 para el factor 3 . Se observa que todos los constructos latentes presentaron valores considerados buenos por la literatura.

\section{Evidencia validez concurrente}

Los resultados de diversas investigaciones evidencian que la ansiedad social involucra expectativas de ser rechazado o de ser evaluado negativamente debido a creencias sobrevaloradas sobre la probabilidad de que ocurra un evento social negativo combinado con una preocupación por la impresión que se genera en la otra persona 
Tabla 2.

Coeficientes de regresión del modelo final

(Baldwin \&

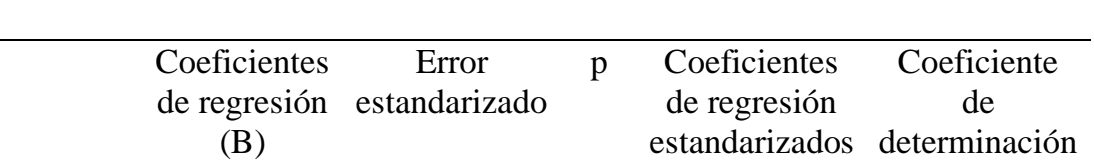

Main, 2001; Smári et al., 1998). Teniendo en cuenta esto, se correlacionaron las

\begin{tabular}{|c|c|c|c|c|c|}
\hline & & & & $(\beta)$ & (R2) \\
\hline Item39 & 1.30 & .13 & $* * *$ & .63 & .40 \\
\hline Item4 & 1.33 & .15 & $* * *$ & .58 & .33 \\
\hline Item 24 & 1.08 & .12 & $* * *$ & .43 & .18 \\
\hline Item35 & 1.43 & .15 & $* * *$ & .65 & .43 \\
\hline Item44 & 1.14 & .13 & $* * *$ & .57 & .32 \\
\hline Item22 & 1.37 & .14 & $* * *$ & .63 & .40 \\
\hline Item 26 & 1.09 & .12 & $* * *$ & .54 & .29 \\
\hline Item 25 & 1.02 & .12 & $* * *$ & .49 & .24 \\
\hline Item 42 & 1.16 & .13 & $* * *$ & .55 & .30 \\
\hline Item30 & 1.18 & .13 & $* * *$ & .57 & .32 \\
\hline Item43 & 1.57 & .15 & $* * *$ & .75 & .56 \\
\hline Item37 & 1.35 & .14 & $* * *$ & .62 & .38 \\
\hline Item41 & 1.38 & .15 & $* * *$ & .59 & .35 \\
\hline Item27 & 1.00 & & & .51 & .26 \\
\hline Item38 & 1.51 & .13 & $* * *$ & .75 & .56 \\
\hline Item13 & 1.11 & .11 & $* * *$ & .56 & .31 \\
\hline Item 28 & 1.32 & .12 & $* * *$ & .69 & .48 \\
\hline Item 23 & 1.23 & .10 & $* * *$ & .76 & .58 \\
\hline Item14 & 1.15 & .11 & $* * *$ & .58 & .34 \\
\hline Item7 & 1.05 & .10 & $* * *$ & .62 & .38 \\
\hline Item2 & 1.00 & & & .59 & .35 \\
\hline Item11 & 1.11 & .13 & $* * *$ & .52 & .27 \\
\hline Item9 & 0.83 & .11 & $* * *$ & .45 & .20 \\
\hline Item17 & 1.11 & .13 & $* * *$ & .57 & .32 \\
\hline Item8 & .98 & .12 & $* * *$ & .51 & .26 \\
\hline Item36 & 1.20 & .10 & $* * *$ & .60 & .36 \\
\hline Item10 & 1.00 & & & .56 & .31 \\
\hline
\end{tabular}
diferentes subescalas del TAS- U y los factores del ERS utilizando el coeficiente de correlación de Pearson y los criterios de Cohen para evaluar los tamaños del efecto (TE). En este caso se considera un TE pequeño cuando la correlación se encuentra por debajo de .10 , medio cuando está en torno a $.30 \mathrm{y}$ grande cuando es mayor que $\quad .50$ (Cohen,1988; Lipsey \& Wilson, 2001).

Como resultado se observaron relaciones positivas significativas (valores $r$ comprendidos entre .03 y .28). Los tamaños del efecto fueron pequeños y nulos entre el factor ansiedad social en situaciones académicas y los factores de expectativas de rechazo social ante personas que no forman parte de la red social constituida por las personas. En el resto de las relaciones, los tamaños del efecto fueron medianos (Tabla 3). 
Tabla 3.

Coeficientes de correlación entre Expectativas de Rechazo Social y Ansiedad Social

\begin{tabular}{lrrr}
\hline & $\begin{array}{c}\text { ERS Vínculos } \\
\text { establecidos }\end{array}$ & $\begin{array}{c}\text { ERS Nuevos } \\
\text { vínculos }\end{array}$ & $\begin{array}{c}\text { ERS Desconocidos } \\
\text { y lugares públicos }\end{array}$ \\
\hline AS Conocidos & $.22^{* *}$ & $.28^{* *}$ & $.24^{* *}$ \\
AS Situaciones Académicas & $.14^{* *}$ & $.09^{*}$ & .03 \\
AS Situaciones Generales & $.21^{* *}$ & $.22^{* *}$ & $.21^{* *}$ \\
AS Abordaje Afectivo- Sexual & $.25^{* *}$ & $.15^{* *}$ & .05 \\
\hline$* * \mathrm{p}<.01 ; * \mathrm{p}<.05$ & & &
\end{tabular}

\section{Discusión}

El establecimiento de relaciones sociales satisfactorias es un factor salugénico y de protección frente a los diferentes factores de estrés psicosocial que las personas afrontan diariamente. Por esto, el estudio del comportamiento interpersonal y de las diferentes variables predictoras de la competencia social resulta de suma importancia en la actualidad. No obstante, para contribuir a este campo de investigación, se hace imprescindible contar con instrumentos de evaluación que sean válidos y confiables. Por ello, el objetivo de este estudio fue la construcción y validación de la Escala de Expectativas de Rechazo Social (ERS).

Se realizó una exhaustiva revisión de escalas que evalúan constructos afines, los desarrollos teóricos y sus recomendaciones para la construcción de escalas, y estudios empíricos antecedentes. Para el diseño se tuvieron en cuenta diferentes situaciones sociales, distintos números de integrantes y diversos niveles de familiaridad. El segundo paso fue recabar evidencia de contenido, a través del juicio de expertos sobre la inclusión y calidad de los reactivos obteniéndose un instrumento inicial de 45 ítems que fueron sometidos a estudios de estructura interna mediante AFE y AFC. Este análisis permitió identificar una estructura interna compuesta de 27 ítems distribuidos en tres factores.

El primer factor se denomina Expectativas de rechazo en situaciones con vínculos establecidos, el cual evalúa expectativas en situaciones cotidianas de interacción con amigos, colegas y familiares. Incluye expresar ideas, hacer propuestas, hacer pedidos y ser integrado en actividades sociales. Los ítems que forman parte de esta subescala también han sido considerados en el contenido de otras escalas afines, tales como la Escala de Miedo a la Evaluación Negativa (FNE de Collins, et al., 2005; Leary, 1983), la escala de Sensibilidad al Rechazo (Mehrabian, 1976), el Cuestionario de evaluación de dificultades interpersonales en la adolescencia (Inglés et al., 2000) y el TAS-U. La 
característica distintiva de los ítems de este factor es el hecho de que los interlocutores son personas con quienes la persona ya ha establecido un vínculo y posee información previa acerca de los posibles resultados de la interacción. Por otro lado, las actividades o demandas son relacionadas a contextos conocidos por la persona y específicas de los mismos (por ejemplo, relativas al lugar de trabajo), y si bien existe cierto grado de incertidumbre en todas las relaciones, afrontar algo nuevo o desconocido puede ser generador de mayores niveles de ansiedad (Gudykunst \& Shapiro, 1996).

El segundo factor se denomina Expectativas de rechazo en situaciones de establecimiento de nuevos vínculos, y evalúa expectativas en situaciones de interacción con personas nuevas que generan algún tipo de interés, ya sea amistoso, académico o sexual por lo cual se incluyen situaciones de abordaje afectivo-sexual. El contenido específico de este factor se vincula a situaciones donde la persona tiene un interés genuino en ser evaluado positivamente o aceptado por su interlocutor, sin tener información previa específica de los posibles resultados. Como afirman McClure et al. (2010), en condiciones óptimas los seres humanos son capaces de equilibrar el deseo de entablar una relación con el miedo al rechazo, así como realizar un acercamiento selectivo a personas que puedan corresponder el interés disminuyendo las posibilidades de ser rechazado, disminuyendo las probabilidades de fracaso interpersonal a un mínimo. Sin embargo, la estrategia utilizada y la percepción de las posibilidades de éxito relacional se basan en la forma en que la persona sopesa el valor del éxito contra el costo de un posible fracaso. En este sentido, personas cuyas expectativas en este tipo de situaciones sean predominantemente de rechazo, posiblemente tendrán conductas evitativas (Gilbert, 2001; Gudykunsta \& Nishida, 2001), disminuyendo sus oportunidades relacionales (Vorauer \& Ratner, 1996). Cabe mencionar que el contenido de este factor se vincula con el de las subescalas de diferentes instrumentos que evalúan constructos interpersonales tales como el Cuestionario de Habilidades Sociales (Morán et al., 2015), la Escala de Autoeficacia Social (Olaz, 2012), el Cuestionario de Ansiedad Social para Adultos (CASO- A30, Caballo et al. 2010) y el TAS-U. Por otro lado, el contenido de algunos de sus ítems también se relaciona con los reactivos del FNE.

El tercer factor se denomina Expectativas de rechazo en lugares públicos y ante desconocidos y el mismo también ha sido evidenciado en la estructura factorial del TASU, el CASO- A30, y la escala de Ansiedad ante la interacción social y ser observado por otros (Heimberg et al., 1993). Este factor evalúa expectativas en situaciones de interacción con personas desconocidas en lugares neutrales y concurridos, como por 
ejemplo un banco. Incluye iniciar conversaciones, compartir espacios y hacer pedidos. Si bien exponerse a una audiencia, iniciar una conversación casual, o hacer una petición, son situaciones que implican un riesgo menor de fracaso o de impacto de sus consecuencias si son ante desconocidos, existen personas que en base a sus expectativas tienden a sobreestimar la probabilidad y el efecto de ser evaluado negativamente. Sumado a ello, los resultados de diferentes investigaciones evidencian que este tipo de individuos tienden a padecer fobia social (Nelson et al., 2010). Por último, es importante destacar que este factor se diferencia del primer factor en base a que los niveles de incertidumbre son mayores cuando nos relacionamos con personas desconocidas que cuando nos comunicamos con miembros de nuestros propios grupos (Gudykunst \& Shapiro, 1996).

Con respecto a la fiabilidad de la ERS, todos los factores presentaron una consistencia interna adecuada (coeficientes alfa de Cronbach y fiabilidad compuesta superiores a .70), según los criterios sugeridos por la preceptiva psicométrica (Nunnally \& Berstein, 1955 citados en Carretero Dios \& Pérez, 2005).

Finalmente se obtuvo evidencia de validez con variables externas corroborando que las expectativas de rechazo social se relacionan significativamente de manera positiva con la ansiedad social. Estos resultados son coincidentes con la literatura e investigaciones antecedentes. Spence, Donovan y Brechman Toussaint (1999) demostraron que los niños con fobia social, tendían a anticipar resultados negativos de las situaciones sociales, a evaluar su propio desempeño más negativamente, y a presentar un mayor nivel de cogniciones negativas en tareas socio-evaluativas en comparación con niños no ansiosos. Alfano, Beidel y Turner (2006) también encontraron que los adultos que presentan mayores niveles de ansiedad social tienen mayores expectativas de rechazo o de resultados negativos sobre su desempeño. Rapee y Heimberg (1997) postulan que, para reducir el riesgo potencial de rechazo en una interacción social, las personas socialmente ansiosas emplean conductas tales como evitar el contacto visual, reducir la producción verbal, utilizar tono de voz bajo o mantenerse aislado dentro de un grupo. Si bien estos comportamientos son sutiles, en la mayoría de los casos no pasan desapercibidos y tienen el efecto de reducir la calidad del desempeño social tornándose una profecía autocumplida, ya que el sujeto recibe feedback tanto de su propio sistema de autovigilancia, como de las respuestas verbales y no verbales de la audiencia, confirmando su inferior rendimiento. De esta manera, aun presentando un adecuado repertorio de habilidades sociales, la persona podría no actuar competentemente debido a una inhibición mediada por expectativas de rechazo y baja autoeficacia social. 
Para finalizar, cabe mencionar que este trabajo presentó algunas limitaciones. En primer lugar, el método de muestreo fue de tipo no probabilístico lo que conlleva un riesgo importante de que los individuos seleccionados no sean representativos de la población. No obstante, se tomaron ciertos recaudos que permitieran reducir la probabilidad de sesgos, entre ellos se incluyeron universidades públicas y privadas, carreras de diferentes disciplinas, y alumnos de distintos años de cursado. Otra limitación a tener en cuenta es que la muestra utilizada en el AFC no se adecuó a los criterios muestrales exigidos por Jackson (2003) que indican una proporción de entre 10 y 20 casos por parámetro, aunque si se tuvieron en cuenta los postulados de otros autores que sugieren trabajar con muestras mayores a 200 casos (Boomsma \& Hoogland, 2001).

En base a los resultados obtenidos en los estudios psicométricos realizados sobre el ERS, se puede concluir que es un instrumento inicialmente válido y confiable para la evaluación de las expectativas de rechazo social en población adulta argentina. En este sentido, resulta una herramienta a considerar para su uso en investigación y diagnóstico. Sin embargo, se considera pertinente ampliar el estudio realizando análisis que permitan obtener más evidencia de estas propiedades, entre ellos, análisis discriminantes, por ejemplo, con medidas de autoestima o autoeficacia, análisis de detección de cambios ante una intervención, y estudios de diferencias de grupos con poblaciones clínicas. 


\section{Referencias}

Alfano, C. A., Beidel, D. C., \& Turner, S. M. (2006). Cognitive Correlates of Social Phobia Among Children and Adolescents. Journal of Abnormal Child Psychology, 34(2), 182-194. https://doi.org/10.1007/s10802-005-9012-9

American Educational Research Association, American Psychological Association, \& National Council of Measurement in Education (2014). The standards for educational and psychological testing. AERA.

Ayduk, O., Downey, G., \& Kim, M. (2001). Rejection sensitivity and depressive symptoms in women. Personality and Social Psychology Bulletin, 27(7), 868-877. https://doi.org/10.1177/0146167201277009

Baldwin, M. W., \& Dandeneau, S. D. (2006). Understanding and Modifying the Relational Schemas Underlying Insecurity. In M.W. Baldwin (Ed.), Interpersonal cognition, (pp. 32-61). Guilford Press.

Baldwin, M.W., \& Main, K. J. (2001). Social anxiety and the cued activation of relational knowledge. Personality and Social Psychology Bulletin, 27(12), 1637-1647. https://doi.org/10.1177/01461672012712007

Boomsma, A.,\& Hoogland, J. J. (2001). The robustness of LISREL modeling revisited. En: R. Cudeck, S. Du Toit, D. Sörbom (Eds). Structural equation modeling: present and future. A festschrift in honor of Karl Jöreskog. p. 139-68. Scientific Software International.

Bretherton, I. \& Munholland, K.A. (1999). Internal working models in attachment relationships. En J. Cassidy \& P.R. Shaver (Eds.), Handbook of attachment: Theory, research, and clinical applications (pp. 89-113). Guilford Press.

Brown, T. A. (2006). Confirmatory factor analysis for applied research. Guilford Press.

Caballo, V; Irurtia, M.A. \& Salazar I. (2011). Abordagem cognitiva na avaliacao e intervencao sobre habilidades socias. En A. Del Prette \& Z.A.P. Del Prette (Orgs.). Psicologia das habilidades sociais: Diversidade teórica e suas implicações (2da Ed) (pp. 67-107). Vozes.

Caballo, V. E., Salazar, I. C., Arias, B., Irurtia, M., Calderero, M., \& CISO-A Research Team. (2010). Validación del Cuestionario de Ansiedad Social para Adultos' (CASO-A30) en universitarios españoles: similitudes y diferencias entre carreras universitarias y comunidades autónomas. Behavioral Psychology, 18(1), 5-34. https://bit.ly/3gxclYV

Carretero- Dios, H., \& Pérez, C. (2005). Normas para el desarrollo y revisión de estudios instrumentales. International Journal of Clinical and Health Psychology, 5 (3), 521-551. https://bit.ly/2zLfhAy

Catell, R. (1966). The Scree test for the number of factors. Multivariate Behavior Research, 1, 245-276. https://doi.org/10.1207/s15327906mbr0102_10

Caouette, J. D., \& Guyer, A. E. (2016). Cognitive distortions mediate depression and affective response to social acceptance and rejection. Journal of affective disorders, 190, 792-799. https://doi.org/10.1016/j.jad.2015.11.015

Cohen, J. (1988). Statistical power analysis for the behavioral sciences (2nd ed.). Erlbaum.

Collins, K. A., Westra, H. A., Dozois, D. J., \& Stewart, S. H. (2005). The validity of the brief version of the Fear of Negative Evaluation Scale. Journal of anxiety disorders, 19(3), 345-359. https://doi.org/10.1016/j.janxdis.2004.02.003

Costello, A. B., \& Osborne, J. W. (2005). Best Practices in Exploratory Factor Analysis: Four Recommendations for Getting the Most From Your Analysis. Assessment, 10 (7), 1-9. https://doi.org/10.4135/9781412995627.d8 
Downey, G., \& Feldman, S. I. (1996). Implications of rejection sensitivity for intimate relationships. Journal of personality and social psychology, 70(6), 1327-1343. https://doi.org/10.1037//0022-3514.70.6.1327

Downey, G., Feldman, S., Khuri, J., \& Friedman, S. (1994). Maltreatment and child depression. In W. M. Reynolds \& H. E Johnson (Eds.), Handbook of depression in childhood and adolescence (pp. 481-508). Plenum.

Driscoll, R. L., Barclay, P., \& Fenske, M. J. (2017). To be spurned no more: The affective and behavioral consequences of social and nonsocial rejection. Psychonomic bulletin \& review, 24(2), 566-573. https://doi.org/10.3758/s13423-016-1114-6

Fiedler, D. \& Beach, L, R. (1978). On the decision to be assertive. Journal of Consulting and Clinical Psychology, 46(3), 537-54. https://doi.org/10.1037/0022006X.46.3.537

Gilbert, P. (2001). Evolution and social anxiety: The role of attraction, social competition, and social hierarchies. Psychiatric Clinics of North America, 24(4), 723-751. https://doi.org/10.1016/S0193-953X(05)70260-4

Gudykunst, W. B., \& Nishida, T. (2001). Anxiety, uncertainty, and perceived effectiveness of communication across relationships and cultures. International journal of Intercultural Relations, 25(1), 55-71. https://doi.org/10.1016/s01471767(00)00042-0

Gudykunst, W. B., \& Shapiro, R. (1996). Communication in everyday interpersonal and intergroup encounters. International Journal of Intercultural Relations, 20, 19-45. https://doi.org/10.1016/0147-1767(96)00037-5

Hair, J. F., Black, W. C., Babin, B. J., Anderson, R. E., \& Tatham, R. L. (2006). Multivariate data analysis (6th Edition). Pearson Education.

Heimberg, R. G., Mueller, G. P., Holt, C. S., Hope, D. A., \& Liebowitz, M. R. (1993). Assessment of anxiety in social interaction and being observed by others: The Social Interaction Anxiety Scale and the Social Phobia Scale. Behavior Therapy, 23(1), 53-73. https://doi.org/10.1016/S0005-7894(05)80308-9

Horn, J. (1965). A rationales and test for the number of factor in factor analysis. Psychometrika, 30, 179-185. https://doi.org/10.1007/bf02289447

Inglés, C. J., Méndez, F. X., \& Hidalgo, M. D. (2000). Cuestionario de evaluación de dificultades interpersonales en la adolescencia. Psicothema, 12, 390-398. https://doi.org/10.1037/t03602-000

Leary, M. R. (1983). A brief versión of the Fear of Negative Evaluation Scale. Personality and Social Psychology Bulletin, 9, 371-375.

Leary, M. R (1986). The impact of interactional impediments on social anxiety and selfpresentation. Journal of Experimental Social Psychology, 22, 122-135. https://doi.org/10.1016/0022-1031(86)90032-6

Leary, M.R., Twenge, J.M. \& Quinlivan, E. (2006). Interpersonal Rejection as a Determinant of Anger and Aggression. Personality and Social Psychology Review, 10(2), 111-132. https://doi.org/10.1207/s15327957pspr1002_2

Lee, C. S., \& Hayes-Skelton, S. A. (2017). Social Cost Bias, Probability Bias, and SelfEfficacy as Correlates of Behavioral Action in Social Anxiety. Behavior Modification, 1-21. https://doi.org/10.1177/0145445517720447

Lent, R.W., \& Brown, S.D. (2006). On conceptualizing and assessing social cognitive constructs in career research: A measurement guide. Journal of Career Assessment, 14(1), 12-35. https://doi.org/10.1177/1069072705281364

Lipsey, M. W., \& Wilson, D. B. (2001). Practical meta-analysis. Sage publications. 
MacDonald, G., \& Leary, M. R. (2005). Why does social exclusion hurt? The relationship between social and physical pain. Psychological bulletin, 131(2), 202. https://doi.org/10.1037/0033-2909.131.2.202

McClure, M. J., Lydon, J. E., Baccus, J. R., \& Baldwin, M. W. (2010). A signal detection analysis of chronic attachment anxiety at speed dating: Being unpopular is only the first part of the problem. Personality and Social Psychology Bulletin, 36(8) 1024-1036. https://doi.org/10.1177/0146167210374238

Mehrabian, A. (1970). The development and validation of measures of affiliative tendency and sensitivity to rejection. Educational and Psychological Measurement, 30(2), 417-428. https://doi.org/10.1177/001316447003000226

Mehrabian, A. (1976). Questionnaire measures of affiliative tendency and sensitivity to rejection. $\quad$ Psychological Reports, $\quad 38, \quad 199-209$. https://doi.org/10.2466/pr0.1976.38.1.199

Merino Soto, C., \& Livia Segovia, J. (2009). Intervalos de confianza asimétricos para el índice la validez de contenido: Un programa Visual Basic para la V de Aiken. Anales de Psicología, 25(1), 169-171. https://bit.ly/3ccrhbt

Morán, V., Olaz, F. Perez, E. R., \& Del Prette. Z. A. P. (2018a). Emotional-Evolutional Model of Social Anxiety in University Student. International Journal of Psychology and Psychological Therapy, 18(3), 315-330. https://bit.ly/3ew6HEz

Morán, V. E., Olaz, F. O., Pérez, E. R., \& Del Prette, Z. A. P. (2018b). Desarrollo y validación del Test de Ansiedad Social para estudiantes universitarios (TAS-U). Liberabit: Revista Peruana de Psicología, 24(2), 195-212. https://doi.org/10.24265/liberabit.2018.v24n2.03

Morán, V. E., Olaz, F. O., \& Del Prette, Z. A. (2015). Social Skills Questionnaire for Argentinean College Students (SSQ-U) Development and Validation. The Spanish Journal of Psychology, 18, E95. https://doi.org/10.1017/sjp.2015.92

Nelson, E. A., Lickel, J. J., Sy, J. T., Dixon, L. J., \& Deacon, B. J. (2010). Probability and cost biases in social phobia: nature, specificity, and relationship to treatment outcome. Journal of Cognitive Psychotherapy, 24(3), 213-228. https://doi.org/10.1891/0889-8391.24.3.213

Olaz, F.O. (2011). Contribucoes da Teoría Social Cognitiva de Bandura para o Treinamento de Habilidades Sociais. En A. Del Prette \& Z.A.P. Del Prette (Orgs.). Psicologia das habilidades sociais: Diversidade teórica e suas implicações (2da Ed.) (pp. 109-148). Vozes.

Olaz, F.O. (2012) Escala de Autoeficacia Social para Universitarios (EAS-U) Estudios Psicométricos y Bases Conceptuales. Editorial Académica Española.

Pajares, F., \& Olaz, F. (2008). Teoria Social Cognitiva e auto-eficacia: uma visão geral. En A. Bandura, R.G. Azzi \& S. Polydoro (Eds), Teoría Social Cognitiva. Conceitos básicos (pp. 97-114). Artmed.

Rapee, R. M. \& Heimberg, R. G. (1997). A cognitive-behavioral model of anxiety in social phobia. Behaviour Research and Therapy, 35, 741-756. https://doi.org/10.1016/s0005-7967(97)00022-3

Smart Richman, L., \& Leary, M. R. (2009). Reactions to discrimination, stigmatization, ostracism, and other forms of interpersonal rejection: a multimotive model. Psychological review, 116(2), 365. https://doi.org/10.1037/a0015250

Sokolowski, K., Schmalt, H.-D., Langens, T. A., \& Puca, R. M. (2000). Assessing achievement, affiliation, and power motives all at once- the Multi-Motive Grid (MMG).Journal of Personality Assessment, 74, 126-145. https://doi.org/10.1207/s15327752jpa740109 
Spence, S. H., Donovan, C., \& Brechman-Toussaint, M. (2000). The treatment of childhood social phobia: The effectiveness of a social skills training-based, cognitive-behavioural intervention, with and without parental involvement. Journal of Child Psychology and Psychiatry, 41(6), 713-726. https://doi.org/10.1017/s0021963099005934

Stephan, W., \& Stephan, C. (1985). Intergroup anxiety. Journal of Social Issues, 41, 15166. https://doi.org/10.1037/t54598-000

Tabak, B. A., Meyer, M. L., Dutcher, J. M., Castle, E., Irwin, M. R., Lieberman, M. D., \& Eisenberger, N. I. (2016). Oxytocin, but not vasopressin, impairs social cognitive ability among individuals with higher levels of social anxiety: A randomized controlled trial. Social cognitive and affective neuroscience, 41(2), 134. https://doi.org/10.1093/scan/nsw041

Tevez Carranza, F. N., Olaz, F. O., \& Moran, V. E. (2018). Expectativas de rechazo social en personas trans y cisgenero. En N. Barqui, G. Genise \& D. Tolosa. Manual integrador hacia la despatologizacion de las indentidades trans. Akadia.

Tobia, V., Riva, P., \& Caprin, C. (2017). Who are the children most vulnerable to social exclusion? The moderating role of self-esteem, popularity, and nonverbal intelligence on cognitive performance following social exclusion. Journal of abnormal child psychology, 45(4), 789-801. https://doi.org/10.1007/s10802-016$\underline{0191-3}$

Vorauer, J. D., \& Ratner, R. K. (1996). Who's going to make the first move? Pluralistic ignorance as an impediment to relationship formation. Journal of Social and Personal Relationships, 13, 483-506. https://doi.org/10.1177/0265407596134001

Wirth, J. H., Bernstein M. J., Wesselmann, E. D. \& LeRoy A. S. (2015). Social cues establish expectations of rejection and affect the response to being rejected. Group Processes \& Intergroup Relations, 1-20. https://doi.org/10.1177/1368430215596073 